[0212-7199 (2005) 22: 10; pp 469-472] ANALES DE MEDICINA INTERNA Copyright (C) 2005 ARAN EDICIONES, S.L.

AN. MED. INTERNA (Madrid) Vol. 22, N. ${ }^{\circ} 10$, pp. 469-472, 2005

\section{Estimación del consumo de suplementos vitamínicos y minerales dispensados a través de las oficinas de farmacia de la provincia de Las Palmas}

\author{
M. D. ALMAZÁN LA-CAVE, P. SAAVEDRA SANTANA, P. LAÍNEZ SEVILLANO, \\ M. C. NAVARRO RODRÍGUEZ, M. MARRERO MONTELONGO, M. TORRES \\ GARCÍA, L. SERRA MAJEM, M. SOSA HENRÍQUEZ
}

Grupo de investigación de Educación y Promoción de la Salud. Universidad de Las

Palmas de Gran Canaria

ESTIMATION OF VITAMIN SUPPLEMENTS AND MINERALS CONSUMPTION DISPENSED THROUGH PHARMACY OFFICES IN THE PROVINCE OF LAS PALMAS

\section{RESUMEN}

Fundamento: El consumo de suplementos polivitamínicos y minerales (SPM) está aumentado en las sociedades occidentales como consecuencia de la preocupación de la población por la salud.

Objetivos: Tener una primera aproximación al consumo de SPM en la provincia de Las Palmas en los 2000 y 2001. Identificar las proporciones de SPM que son dispensados como especialidades farmacéuticas y los que lo hacen como productos de parafarmacia. Describir cual es la tendencia evolutiva en el consumo de estos SPM en el período de un año.

Método: La información se obtuvo de los listados de ventas de todas las especialidades farmacéuticas y productos de parafarmacia de los años 2000 y 2001, que contienen en su composición al menos una vitamina y/o un mineral.

Resultados: habitualmente se dispensan 297 especialidades farmacéuticas y 216 productos de parafarmacia. Las especialidades farmacéuticas constituyeron el $65,6 \%$ de las ventas y entre ellas, las vitaminas resultaron ser las más dispensadas (41,5\%). En las especialidades de parafarmacia, las más vendidas fueron los compuestos de vitaminas y minerales $(34,6 \%)$.

Con respecto al año 2000, en el año 2001 se produjo un aumento estadísticamente significativo en el consumo de productos de parafarmacia, permaneciendo estable el de las especialidades farmacéuticas.

Conclusión: en base a las ventas realizadas, en la provincia de Las Palmas el consumo de SPM parece realizarse mayoritariamente por medio de especialidades farmacéuticas y en menor grado mediante productos de parafarmacia. Sin embargo, en el período 2000-2001, se observó un incremento del consumo sólo en los productos de parafarmacia.

PALABRAS CLAVES: Suplementos vitamínicos. Minerales. Parafarmacia. Consumo. Farmacia. Las Palmas.

\section{ABSTRACT}

Background: Vitamin supplements and minerals consumption (SPM) is increasing in occidental societies due to the growing concern about health by the population

Objectives: To have a initial approaching to the to SPM consumption in the Province of Las Palmas through 2000 and 2001. To identify SPM proportions that are dispensed as pharmaceutical specialities and those who are sold as parapharmacy products. Finally, to describe the evolution of this consumption throughout a year

Method: The information was obtained through the list of the whole pharmaceutical specialities and parapharmacy products through 200 and 2001 who have at least a vitamin in its composition and/or a mineral.

Results: Usually, 297 pharmaceutical specialities and 216 parapharmacy products are currently being sold. Pharmaceutical specialities comprised $65.6 \%$ of the whole products sold and within them, vitamins were the most dispensed (41.5\%). Regarding parapharmacy products, vitamins and minerals compounds were the products more sold (34.6\%).

Comparing to 2000, during 2001 there was a statistically significant increase in the consumption of parapharmacy products, remaining without changes the use of pharmaceutical specialities.

Conclusion: On the basis of sold XX SPM consumption seems to be due mainly by pharmaceutical specialities rather than parapharmacy products. Nevertheless through 2001 there was an increase only in the parapharmacy products.

KEY WORDS: Vitamins supplements. Minerals. Parapharmacy. Consumption. Pharmacy. Las Palmas.

Almazán La-Cave MD, Saavedra Santana P, Lainez Sevillano P, Navarro Rodríguez MC, Marrero Montelongo M, Torres García M, Serra Majem L, Sosa Henríquez. M. Estimación del consumo de suplementos vitamínicos y minerales dispensados a través de las oficinas de farmacia de la provincia de Las Palmas. An Med Interna (Madrid) 2005; 22: 469-472.

\section{INTRODUCCIÓN}

En los últimos años se ha incrementado el interés de la población por la relación existente entre la alimentación y la salud, de tal manera que el concepto clásico de "nutrición adecuada", entendido como el aporte de nutrientes requeridos para satisfacer las necesidades del organismo, está siendo sustituido por el concepto de "nutrición óptima", en donde además se contempla la potencialidad de los alimentos para promocionar la salud $(1,2)$.

Esto ha condicionado la aparición en el mercado de un nuevo y amplio grupo de productos, situados entre los medi-

Trabajo aceptado: 10 de junio de 2005

Correspondencia: M ${ }^{\mathrm{a}}$ Carmen Navarro Rodríguez. Universidad de Las Palmas de Gran Canaria. Facultad de Ciencias de la Salud. Apartado 550. 35080 Las Palmas de Gran Canaria. Fax: 928451 428. e-mail: mnavarro@denf.ulpgc.es 
camentos y los alimentos. Entre ellos se encuentran los alimentos funcionales, los alimentos enriquecidos y los complementos alimenticios entre los que incluiríamos a los suplementos polivitaminicos minerales (SPM). Existen estudios que ponen de manifiesto que los SPM aportan ventajas sanitarias específicas. Así, se han asociado a una disminución del riego de tener descendientes con malformaciones congénitas (3) y de padecer enfermedades neoplásicas $(4,5)$, cardiovasculares (6-8), cataratas y otros procesos degenerativos oculares (9-10).

Estos posibles efectos beneficiosos de los SPM, unido al hecho observado de una mayor preocupación de la población por la salud y a la necesidad de la industria alimentaria y farmacéutica de ampliar su mercado, ha conducido a un cambio importante en el consumo de suplementos polivitamínicos minerales. Así, se ha pasado de una situación basada en el establecimiento de las ingestas recomendadas, para prevenir las deficiencias de vitaminas y minerales (11) a otra en la que lo que se requiere es la necesidad de definir las dosis máximas autorizadas de estos principios alimentarios.

Hemos realizado el presente estudio, como una primera aproximación al conocimiento de la realidad del consumo de SPM en nuestra provincia.

\section{MÉTODO}

Para la realización de este trabajo hemos consultado los listados de las ventas de la Cooperativa Farmacéutica Canaria en la provincia de Las Palmas, correspondiente a los años 2000-2001, de todas las especialidades farmacéuticas y productos de parafarmacia que contienen en su composición al menos una vitamina y/o un mineral. El número total de productos estudiados fue de 513, de los cuales 297 correspondían a especialidades farmacéuticas y 216 a productos de parafarmacia.

Las presentaciones en donde no se registraron movimientos en los 24 meses correspondientes a los años 2000 y 2001, fueron excluidas del estudio.

Agrupamos las especialidades, de acuerdo con la similitud o semejanza de su composición cualitativa, para así realizar un mejor tratamiento de las mismas.

Clasificamos a todos estos productos en especialidad farmacéutica o producto de parafarmacia, de acuerdo con el catálogo editado por el Consejo General de Colegios Oficiales de Farmacéuticos.

Los datos fueron incluidos en una hoja de cálculo, con el programa informativo Excel de Microsoft-Office-2000. Después fueron exportados al programa estadístico SPSS 11.0. Con el mismo se realizó el análisis descriptivo, mediante la elaboración de un listado de frecuencias de las variables cualitativas y el correspondiente cálculo de las distintas medidas de tendencia central y de dispersión de las variables cuantitativas. También se compararon las medias para muestras independientes de los distintos grupos de medicamentos, tanto dentro de las especialidades farmacéuticas como de los productos de parafarmacia. La significación de las variables cuantitativas fue analizada mediante la prueba de la " $t$ " de Student, estableciendo el nivel de significación en el 5\% ( $\mathrm{p}<$ $0,05)$.

\section{RESULTADOS}

En la tabla I se puede observar la distribución global de los productos estudiados, que fueron 513, de los cuales 297 correspondían a especialidades farmacéuticas y 216 a productos de parafarmacia. Se obtuvieron datos de ventas de 248 especialidades farmacéuticas y de 130 productos de parafarmacia. El resto corresponde a productos que no se comercializan en la provincia.

\section{TABLA I}

DISTRIBUCIÓN DE LAS ESPECIALIDADES FARMACÉUTICAS Y DE PARAFARMACIA

\begin{tabular}{lcccc}
\hline Producto & Consultados & $\%$ & Estudiados & $\%$ \\
\hline Especialidades farmacéuticas & 297 & 57,89 & 248 & 65,61 \\
Parafarmacia & 216 & 42,11 & 130 & 34,39 \\
\hline
\end{tabular}

Especialidades farmacéuticas

Polivitamínicos-minerales

Vitaminas

Hierro-ácido fólico

Minerales

Tónicos

$\begin{array}{cccc}45 & 15,15 & 20 & 8,06 \\ 104 & 35,02 & 103 & 41,53 \\ 48 & 16,16 & 39 & 15,73 \\ 68 & 22,90 & 58 & 23,39 \\ 32 & 10,77 & 28 & 11,29\end{array}$

Parafarmacia

Deportistas

Faneras

Vitaminas-minerales

Jalea real/polen/levadura

Ginseng

\begin{tabular}{rcrc}
29 & 13,43 & 18 & 13,85 \\
31 & 14,35 & 23 & 17,69 \\
78 & 36,11 & 45 & 36,42 \\
60 & 27,78 & 35 & 26,92 \\
18 & 8,33 & 9 & 6,92 \\
\hline
\end{tabular}

Las especialidades farmacéuticas constituyeron el 65,6\% de las ventas, mientras que los productos de parafarmacia supusieron el $34,4 \%$ de las mismas.

Dentro de las especialidades farmacéuticas, los compuestos con polivitaminas y minerales fueron los menos vendidos $(8,06 \%)$ y las vitaminas las más dispensadas $(41,5 \%)$. En las especialidades de parafarmacia, los menos vendidos fueron aquellos que en su composición contienen ginseng y los que más los compuestos de vitaminas y minerales $(34,62 \%)$.

En la tabla II se recoge la evolución de las ventas de las especialidades farmacéuticas y productos de parafarmacia entre los años 2000 y 2001. Los productos de parafarmacia registraron un incremento que fue estadísticamente significativo $(\mathrm{p}=0,004)$, diferencia que no se constató en las especialidades farmacéuticas.

En la tabla III se muestra la evolución de las ventas de los suplementos polivitamínicos minerales englobados dentro de las especialidades farmacéuticas entre el 2000 y el 2001.

TABLA II

EVOLUCIÓN DE LAS VENTAS DE LAS ESPECIALIDADES FARMACÉUTICAS Y PRODUCTOS DE PARAFARMACIA ENTRE 2000 Y 2001

\begin{tabular}{lcc}
\hline & 2000 & 2001 \\
\hline Especialidades farmacéuticas & $77.275,8 \pm 6581,0$ & $79.906,5 \pm 6.291,2$ \\
Productos de parafarmacia & $7.176,3 \pm 944,2$ & $8.314,8 \pm 800,3$ \\
\hline
\end{tabular}


TABLA III

EVOLUCIÓN DE LAS VENTAS DE LOS SUPLEMENTOS POLIVITAMÍNICOS MINERALES ENGLOBADOS DENTRO DE LAS ESPECIALIDADES FARMACÉUTICAS ENTRE 2000 Y 2001

\begin{tabular}{lccc}
\hline Producto & 2000 & 2001 & $p$ \\
\hline Polivitaminas-minerales & $7.647,2 \pm 1307,3$ & $6.886,2 \pm 648,6$ & 0,08 \\
Polivitaminas & $2.048,9 \pm 192,8$ & $1.992,5 \pm 179,3$ & 0,466 \\
Vitaminas A-E & $4.321,0 \pm 589,0$ & $5.618,5 \pm 1054,1$ & 0,001 \\
Vitaminas D y análogos & $1442,1 \pm 98,1$ & $1.491,7 \pm 115,7$ & 0,270 \\
Vitamina B & $9.195,8 \pm 715,9$ & $9.160,0 \pm 780,2$ & 0,908 \\
Vitaminas C-A & $7.925,9 \pm 2509,0$ & $6.863,9 \pm 1772,5$ & 0,244 \\
Otras vitaminas & $2.154,0 \pm 383,5$ & $2.216,6 \pm 539,5$ & 0,746 \\
Productos para la anemia & $22.854,3 \pm 1216,4$ & $24.933,5 \pm 1778,1$ & 0,003 \\
Minerales & $17.110,7 \pm 1685,2$ & $18.293,2 \pm 1277,3$ & 0,06 \\
Tónicos & $4.017,4 \pm 612,8$ & $3.941,6 \pm 389,3$ & 0,721 \\
\hline
\end{tabular}

Se observó un incremento estadísticamente significativo en las ventas de las vitaminas A-E $(\mathrm{p}=0,001)$ y de hierro y ácido fólico $(\mathrm{p}=0,003)$. Pese a no alcanzar significación estadística, estuvieron muy cerca de hacerlo los incrementos observados en la venta de minerales $(\mathrm{p}=0,06)$ y de polivitamínicos y minerales $(\mathrm{p}=0,08)$.

En la tabla IV se recoge la evolución de las ventas de los productos de parafarmacia entre 2000 y 2001. Obtuvimos un incremento estadísticamente significativo en las ventas de vitaminas y minerales ( $\mathrm{p}=0,001)$, así como de los compuestos que contienen jalea real y polen $(p=0,001)$. El descenso en las ventas de levadura no alcanzó el nivel de significación, aunque estuvo muy próximo a ello $(\mathrm{p}=0,07)$.

\section{TABLA IV}

EVOLUCIÓN DE LAS VENTAS DE LOS PRODUCTOS DE PARAFARMACIA ENTRE 2000 Y 2001

\begin{tabular}{lccc}
\hline Producto & 2000 & 2001 & $P$ \\
\hline Deportistas & $1104,0 \pm 126,9$ & $1060 \pm 110,7$ & 0,376 \\
Faneras & $1332,2 \pm 105,8$ & $1408,0 \pm 211,7$ & 0,280 \\
Vitaminas-minerales & $2638,1 \pm 750,9$ & $3687,7 \pm 619,6$ & 0,001 \\
Jalea real-polen & $579,2 \pm 71,7$ & $762,8 \pm 100,4$ & 0,001 \\
Levadura & $730,5 \pm 174,0$ & $613,8 \pm 118,3$ & 0,07 \\
Ginseng & $729,1 \pm 104,9$ & $782,3 \pm 87,9$ & 0,806 \\
\hline
\end{tabular}

\section{DISCUSIÓN}

Es conocido que la ingestión de cantidades adecuadas de vitaminas y minerales es importante en el mantenimiento y promoción de la salud, así como en la prevención de diversas patologías (3 -10).

En España se han realizado en los últimos años varios estudios basados en las encuestas de nutrición realizadas en distintas comunidades autónomas que han evidenciado la existencia de ingestas de riesgo para determinados nutrientes (12), pero menos estudiado ha sido el consumo de suplementos vitamínico-mineral. Con los datos existentes se estima que en España un 16\% de la población consume habitual o cíclicamente estos suplementos, en su mayoría como autoprescrip- ción. Este consumo lo lleva a cabo, bien en forma de multivitaminas $(53 \%)$, minerales $(29 \%)$ o ambos $(3 \%)$ y reconocen ingerirlos, principalmente, para tener más energía (22\%) y para sentirse mejor (18\%). La mayoría consumen los suplementos vitamínico- mineral en forma de cápsulas (65\%), a diario o casi todos los días (13).

En la Comunidad Autónoma Canaria, el consumo de suplementos polivitamínicos minerales se estima según datos aportados por la Encuesta de Nutrición de Canarias en un 6,6\% (14), siendo los suplementos más empleados los compuestos de vitaminas y minerales $(48,4 \%)$, de hierro $(20,4 \%)$, polivitamínicos $(14 \%)$, vitaminas C $(14 \%)$ y calcio $(3,2 \%)$. Además este estudio estableció que durante el año anterior a la realización de dicha Encuesta, el consumo de suplementos fue del $22 \%$. Este resultado es similar al encontrado en los países centroeuropeos $(21 \%)$, superior al descrito en Italia $(10 \%)$ y Francia $(18 \%)$ y notablemente inferior al hallado en los países anglosajones (31\%), nórdicos (33\%) y Estados Unidos $(51 \%)(15,16)$.

Con nuestro trabajo pretendemos contribuir al conocimiento de la situación existente, haciendo una estimación de las ventas, que de este tipo de productos se realizan a través de las Oficinas de Farmacia de la provincia de Las Palmas, apoyándonos en la certeza de que el consumidor habitual de suplementos polivitamínicos minerales en España, sigue recurriendo a la farmacia como principal proveedor de este tipo de productos, seguido con gran diferencia de las tiendas de alimentos dietéticos y otras (17).

Hemos observado que en nuestra provincia el consumo de SPM se realiza sobre todo a través de especialidades farmacéuticas, que recogimos en el 65,6\% de las prescripciones frente al $34,4 \%$ de los productos de parafarmacia. Sin embargo, cuando analizamos su progresión hemos constatado que el consumo de especialidades farmacéuticas ha permanecidos sin cambios estadísticamente significativos entre 2000 y 2001, mientras que el consumo de productos de parafarmacia mostró un incremento que fue estadísticamente significativo.

Al analizar de manera individualizada la evolución de los diferentes productos que hemos englobado como suplementos polivitamínicos minerales entre 2000 y 2001, encontramos que se había producido un incremento en el consumo de vitaminas A y E ( $\mathrm{p}=0,001)$, así como de hierro y ácido fólico ( $\mathrm{p}$ $=0,003$ ). No obtuvimos diferencias en la evolución del consumo de los demás productos, aunque en algunos casos, como en el consumo de minerales y de polivitamínicos minerales el nivel de significación estuvo muy cercano al $5 \%$ previamente establecido como válido.

El incremento del consumo de vitaminas A y E podría deberse a la reciente aparición en el mercado de nuevas presentaciones farmacéuticas que contienen vitamina $\mathrm{E}$ a distintas concentraciones, en sintonía con las nuevas tendencias que le confieren beneficios en la prevención de la enfermedad coronaria (18).

Finalmente hemos apreciado un incremento, estadísticamente significativo, en el consumo de vitaminas y minerales y de jalea real-polen en los productos de parafarmacia.

Dentro de las limitaciones de nuestro estudio, se encuentra en primer lugar la imposibilidad de comparar nuestros resultados con otros trabajos al no haber podido encontrar en la literatura un diseño similar al nuestro, pese a la búsqueda efectuada en el Pub Med. 
Otra limitación es que hemos recogido los productos dispensados a través de la Cooperativa Farmacéutica Canaria, pero no podemos saber si realmente las personas que los han adquirido han terminado consumiéndolos o no y de que manera.

\section{Bibliografía}

1. Ames BN. The metabolic tune-up: metabolic harmony and disease prevention. J Nutr 2003; 133 (Supl. 1): 1544S-8S.

2. MB Silveira Rodríguez, S Monereo Megías y B Molina Baena. Alimentos funcionales y nutrición óptima. ¿cerca o lejos? Rev Esp Salud Pública 2003; 77: 317-31

3. Yang Q, Khoury MJ, Olney RS, Mulinare J. Does periconceptional multivitamin use reduce the risk for limb deficiency in offspring? Epidemiology 1997; 8: 157-61.

4. Grant WB, Garland CF.A critical review of studies on vitamin D in relation to colorectal cancer. Nutr Cancer 2004; 48: 115-23.

5 Patterson RE, Neuhauser ML, White E, Hunt JR, Kristal AR. Cancer related behaviour of vitamin supplement users. Cancer Epidemiol Biomarker P rev 1998; 7: 79-81.

6. Mark SD, Wang W, Fraumeni JF Jr, Li JY, Taylor PR, Wang GQ, et al: Lowered risks of hypertension and cerebrovascular disease after vitamin/mineral supplementation: the Linxian Nutrition Intervention Trial. Am J Epidemiol 1996; 1143: 658-64.

7. Meyer F, Bariah I, Daganais GR. Lower ischemic heart disease incidence and mortality among vitamin supplement users. Can J Cardiol 1996; 12: $930-4$.

8. Holmquist C, Larsson S, Wolk A, de Faire U. Multivitamin supplements are inversely associated with risk of myocardial infarction in men and women-Stockholm Heart Epidemiology Program (SHEEP). J Nutr 2003; 133: 2650-4.

9. Brown NA, Bron AJ, Harding JJ, Dewar HM. Nutrition supplements and the eye. Eye 1998; 12: 127-33.

10. Shimakawa T, Nieto FJ, Malinow MR, Chambless LE, Schreiner PJ,
No obstante, creemos que en líneas generales este trabajo nos ha permitido obtener una primera aproximación al consumo de SPM en nuestra provincia y nos ha puesto de manifiesto un incremento en determinados productos, hallazgos, que en futuros estudios deberán ser confirmados.
Szklo M. Vitamin intake. A possible determinant of plasma homocysteine among middle-aged adults. Ann Epidemiol 1997; 7: 285-93.

11. American Dietetic Association. Recommendations concerning supplement usage: ADA statement. J Am Diet Assoc 1987; 46: 1342-3.

12. Aranceta J, Serra-Majem L, Ortega RM , Entrala A, Gil A. Las vitaminas en la alimentación de los españoles. Estudio eVe. Ed Médica Panamericana 2000.

13. Arteaga MM. Conocimiento de las vitaminas por el consumidor. En: Las vitaminas en la alimentación de los españoles. Estudio eVe. Eds: J. Aranceta, L Serra-Majen, RM Ortega, A Entrala, A Gil.

14. Ed Médica Panamericana 2000: 143-50.

15. Serra-Majem L, Ribas L, Armas A, en nombre del equipo investigador de ENCA. Encuesta Nutricional de Canarias. Ingesta de energía, nutrientes y riesgo de ingestas inadecuadas. Vol 3. Tenerife: Servicio Canario de Salud; 2000.

16. Subar AF, Block G. Use of vitamin and mineral supplements demographics and amounts of nutrients consumed. The 1987 Health Interview Survey. Am J Epidemiol 1990; 132: 1091-101.

17. Gassin AL. Fortification and the European consumer. Consumer awareness and attitudes to food fortification. Scand J Nutrition 1999; 43 (Supl. 35): S122-S124.

18. Serra-Majem L, Ribas L, Sainz P, López M. Consumo de suplementos vitamínicos-minerales en la población española (1996). Nutr Clin 1996; 16: 7-15.

19. Serra-Majem L. la suplementación con vitaminas y minerales. Alimentos nutracéuticos. Nutrición comunitaria. Universidad de Cantabria. 2002: 227-33. 